\title{
Neurotherapeutic and neuroprosthetic effects of implanted functional electrical stimulation for ambulation after incomplete spinal cord injury
}

\author{
Stephanie Nogan Bailey, BSE; ${ }^{1 *}$ Elizabeth C. Hardin, PhD; ${ }^{1}$ Rudi Kobetic, $M S ;^{1}$ Lisa M. Boggs, $^{\text {MPT; }}{ }^{1}$ \\ Gilles Pinault, MD; ${ }^{1}$ Ronald J. Triolo, $\mathbf{P h D}^{1-2}$ \\ ${ }^{1}$ Louis Stokes Cleveland Department of Veterans Affairs Medical Center, Cleveland, OH; ${ }^{2}$ Departments of \\ Orthopaedics and Biomedical Engineering, Case Western Reserve University, Cleveland, $\mathrm{OH}$
}

\begin{abstract}
The purpose of this single-subject study was to determine the neurotherapeutic and neuroprosthetic effects of an implanted functional electrical stimulation (FES) system designed to facilitate walking in an individual with a longstanding motor and sensory incomplete spinal cord injury. An implanted pulse generator and eight intramuscular stimulating electrodes were installed unilaterally, activating weak or paralyzed hip flexors, hip and knee extensors, and ankle dorsiflexors during 36 sessions of gait training with FES. The neurotherapeutic effects were assessed by a comparison of pre- and posttraining volitional walking. The neuroprosthetic effects were assessed by a comparison of posttraining volitional and FESassisted walking. Treatment resulted in significant $(p<0.005)$ volitional improvements in 6-minute walking distance and speed, speed during maximum walk, double support time, and $10 \mathrm{~m}$ walking speed. Posttraining FES-assisted walking resulted in significant additional improvements in all these measures, except $10 \mathrm{~m}$ walking speed. When the subject was using FESassisted gait, maximum walking distance, peak knee flexion in swing, peak ankle dorsiflexion in swing, and knee extension moment also significantly increased. Neuroprosthetic gains were sufficient to enable the subject to advance from household ambulation to limited community ambulation. Additionally, the subject could perform multiple walks per day when using FESassisted gait, which was impossible with volitional effort alone.
\end{abstract}

Key words: functional electrical stimulation, functional outcomes, gait, gait training, neurological gait disorders, neuroprosthetic, neurotherapeutic, rehabilitation, spinal cord injury, stimulation, walk.

\section{INTRODUCTION}

Because of improved vehicular safety, acute trauma care, and innovative medical treatments, the percentage of individuals with incomplete versus complete spinal cord injuries (SCIs) is increasing [1]. Partial paralysis from incomplete SCI limits participation in society and adversely affects general health and well-being [2]. These individuals often remain sedentary and exhibit compromised physical endurance, with limited standing and walking capabilities. Fortunately, rehabilitation interventions can enhance physical limitations, elevate activity level, and improve ambulatory function for these individuals. The interventions can be classified into neurotherapeutic interventions that maximize volitional strength, coordination, and locomotor ability, and neuroprosthetic interventions that augment and enhance baseline voluntary function.

\footnotetext{
Abbreviations: $\mathrm{AFO}=$ ankle-foot orthosis, $\mathrm{ASIA}=$ American Spinal Injury Association, BWSTT = body-weight-supported treadmill training, CWRU = Case Western Reserve University, FES = functional electrical stimulation, OGT = overground gait training, SCI = spinal cord injury, URS = Usability Rating Scale, VA = Department of Veterans Affairs.

*Address all correspondence to Stephanie Nogan Bailey, BSE; Louis Stokes Cleveland Department of Veterans Affairs Medical Center, Motion Study Laboratory 151A-W, 10701 East Boulevard, Cleveland, OH 44106; 216-791-3800, ext 4615; fax: 216-231-3433. Email: sbailey@fescenter.org DOI:10.1682/JRRD.2009.03.0034
} 
One aim of neurotherapeutic interventions is improving volitional walking ability via modalities such as strength and endurance exercise, balance training, overground gait training (OGT), body-weight-supported treadmill training (BWSTT), and functional electrical stimulation (FES) assisted gait training. To maximize the potential impact of these interventions, therapists usually prescribe them within weeks or months of injury. One recent study found that lower-limb strength, walking speed, and distance greatly improved after 12 weeks of therapeutic modalities consisting of OGT or BWSTT in the acute injury phase [3]. Subjects were all initially nonambulatory and made measurable volitional gains by the end of rehabilitation that included a $0.8 \mathrm{~m} / \mathrm{s}$ increase in $15 \mathrm{~m}$ walking speed and a $76 \mathrm{~m}$ increase in the 6-minute walk distance. Similar outcomes were achieved regardless of the neurotherapeutic intervention because both OGT and BWSTT produced equivalent gains in voluntary function. A crossover study demonstrated that the addition of FES to BWSTT could potentially accelerate gait training in subjects with acute injuries [4]. For ambulatory persons with chronic incomplete SCI, the use of surface stimulation during OGT or BWSTT increased volitional muscle strength [5-6], stride length [5,7-8], and walking speed [6-9]. To eliminate the need to properly place surface electrodes daily, subjects can use implanted FES systems for several functional tasks [10-11]. Additionally, subjects prefer using FES systems versus traditional braces during upright activities [10-11].

Neuroprosthetic interventions employ FES to assist tasks such as standing or walking [12]. Such interventions can be effective for acute or chronic SCI regardless of baseline function. For example, one case study documented the deployment of an FES system in a nonambulatory male with a chronic C6 incomplete SCI (American Spinal Injury Association [ASIA] C). He could stand volitionally, but could not initiate a forward step. He received an eight-channel implanted motor neuroprosthesis to facilitate walking by delivering stimulation bilaterally to the hip flexors, knee extensors, and ankle dorsiflexors to assist his gait during the swing and single-limb stance phases [13]. After 12 weeks of OGT with FES, the subject achieved a maximum walking distance of $309 \mathrm{~m}$ and a maximal speed of $0.20 \mathrm{~m} / \mathrm{s}$ with the neuroprosthesis, but volitional walking function was unchanged. No neurotherapeutic effect occurred after this intensive training, and without stimulation he remained nonambulatory. However, the neuroprosthetic effect of the FES system moved him from a nonambulator to a limited community ambulator.
Although neurotherapeutic interventions can produce functional gains, a neuroprosthesis could be a powerful adjunct to traditional physical therapy to further improve ambulatory function above volitional levels. Another reasonable expectation is that an implanted neuroprosthesis could produce changes in maximal volitional function. That is, neuroprosthetic interventions may have neurotherapeutic value. To determine the relative neurotherapeutic and neuroprosthetic effects of an implanted neuroprosthesis, we designed and conducted a single-subject multiple-baseline case study on an ambulatory volunteer with chronic incomplete SCI. The purposes of this single-subject case study were to quantify (1) the neurotherapeutic effects of FESaugmented OGT on volitional gait function and (2) the neuroprosthetic effects of an implanted FES system apart from those volitionally achievable.

\section{METHODS}

After approval by the Institutional Review Board of the Louis Stokes Cleveland Department of Veterans Affairs (VA) Medical Center, a 35-year-old male who was 18 months postinjury was enrolled in the study [13]. He presented with a C6 incomplete SCI (ASIA D) characterized by significant left side weakness, in addition to weakness of his trunk and upper limbs. He was unable to maintain standing balance without arm support on an assistive device and walked only limited distances $(<30 \mathrm{~m})$ with a wheeledwalker and left ankle-foot orthosis (AFO). Ambulatory distance was limited by upper-limb fatigue due to the compensatory strategy of hip and trunk elevation to clear his weaker left leg during swing.

Informed consent was obtained and the following baseline measures were assessed: 6-minute walking distance and speed, maximum walking distance and speed, threedimensional quantitative gait analysis of spatial-temporal and kinematic parameters, peak isokinetic knee extension moment, and manual muscle test scores. The participant then received the Case Western Reserve University (CWRU)/VA neuroprosthesis that has successfully provided exercise, standing, and transfer assistance to volunteers with low cervical- and thoracic-level injuries [13-15]. The CWRU/VA neuroprosthesis consists of an implanted receiver-stimulator, eight surgically implanted intramuscular electrodes, and a wearable external control unit that powers and transmits control information to the implanted receiver-stimulator via a transmitting coil affixed to the skin [16] (Figure 1). 


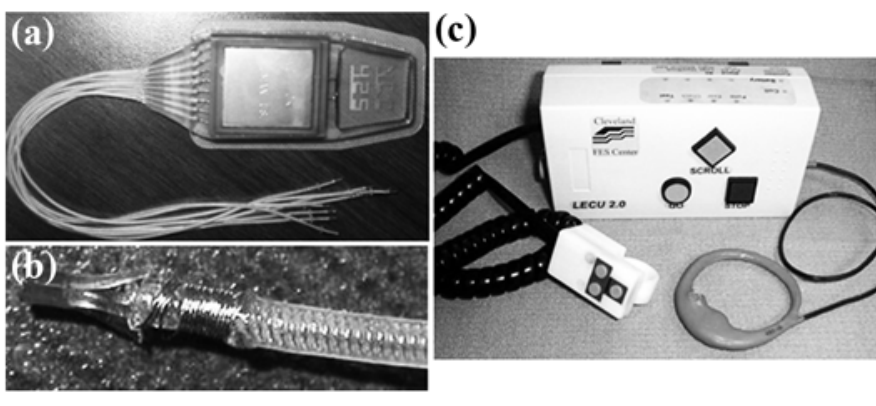

Figure 1.

Neuroprosthesis consists of (a) implanted receiver-stimulator and (b) intramuscular electrodes, along with (c) wearable external control unit, finger switch, and transmitting coil.

The participant had adequate volitional control over his right leg for ambulation; thus, his left leg was implanted with stimulating electrodes to address his primary gait deficits. The implanted muscles were the (1) iliopsoas for hip flexion, (2) tensor fasciae latae for hip flexion and abduction, (3) gluteus medius for hip abduction, (4) posterior portion of adductor magnus for hip extension, (5) gluteus maximus for hip extension, (6) vastus lateralis for knee extension, (7) tibialis anterior for ankle dorsiflexion, and (8) peroneus longus for foot eversion.

After implantation, the participant underwent 6 weeks of limited activity to promote healing and encapsulation of the implanted components before initiating use of the neuroprosthesis. Stimulation patterns modulating pulse width $(0-200 \mu \mathrm{s})$ and frequency $(0-30 \mathrm{~Hz})$ with constant current amplitude (20 mA) were developed for exercise and walking. A home exercise program that included exercises to build the strength and endurance of the stimulated muscles was prescribed and followed. The program consisted of four exercise patterns, each correlating with a phase of gait, providing stimulation to the (1) extensors as a group (stance), (2) flexors as a group (swing), (3) ankle dorsiflexor (swing), and (4) knee extensor (stance and terminal swing). The daily home exercise regimen consisted of 60 minutes of exercise 1 and 3 sets of 10 repetitions for exercises 2, 3, and 4. Compliance with home exercise was monitored by the participant's self-report.

Because the participant could walk volitionally, gait training was started simultaneously with the home exercise program. Initially, the research physical therapist triggered each FES-assisted left step for the participant with a finger switch while the participant learned to coordinate his nonFES right leg. The participant progressed to triggering each step with the finger switch independently and finally to walking with a pattern of continuously cycling stimulation.

Data collection to quantify initial voluntary function was repeated after 36 sessions of FES-assisted gait training. We collected posttraining data both with and without FES to isolate the neurotherapeutic and neuroprosthetic effects. The 6-minute and maximum walking tests were performed in random order on 5 different days with one FES and one nonFES condition for each walking test per day. We separated the four walks by at least an hour to offset any effects of fatigue. Walking distances and speeds were measured with a calibrated distance measuring wheel and a stopwatch.

We analyzed the participant's gait with and without FES on the same day using a motion capture system (Vicon 370; Oxford, United Kingdom) to eliminate variability in marker placement and to minimize laboratory time for the participant. Fifteen markers were placed on the pelvis and legs according to Vicon's Lower Extremity Plug-In Gait model [17], which was used for kinematic calculations. We collected 5 to 10 trials for each condition, providing 5 minutes of rest between trials to minimize fatigue. Double support time, step length, walking speed, peak knee flexion in swing, and peak ankle dorsiflexion in swing were derived from the kinematic data acquired over a $10 \mathrm{~m}$ walkway.

We collected 5 to 10 repetitions of peak voluntary and FES-assisted isokinetic knee extensor moment with a Biodex Pro System 3 dynamometer (Shirley, New York). While sitting erect, the subject was instructed to exert maximal effort to extend his knee from $90^{\circ}$ of flexion to full extension while the dynamometer maintained a constant angular velocity of $30^{\circ}$. One minute of rest was provided between trials, and we collected data with and without FES on the same day to ensure consistent lowerlimb and trunk positioning in the apparatus. In addition, manual muscle test scores were collected with and without stimulation on the same day for the five muscle groups that the lower-limb ASIA motor test comprises: hip flexors, knee extensors, ankle dorsiflexors, long toe extensors, and ankle plantar flexors.

We applied paired $t$-tests with Bonferroni correction $(p<0.005)$ to the data to determine (1) the effects of exercise and gait training on voluntary walking function between baseline (pre-FES) and postrehabilitation measures and (2) the significance of the neuroprosthetic effects, which were evident when we compared performance with and without FES at end of treatment. In this way, we could determine the incremental effects of FES-assisted gait on walking performance, in addition to maximal voluntary function postrehabilitation. 


\section{RESULTS}

The statistical analyses revealed the key outcome factors for either of the modalities to be (1) 6-minute walk distance, (2) speed during 6-minute walk, (3) maximum walk distance, (4) speed during maximum walk, (5) double support time, (6) $10 \mathrm{~m}$ walking speed, (7) peak knee flexion in swing, (8) peak ankle dorsiflexion in swing, and (9) peak isokinetic knee extension moment. Although a strong trend emerged toward increased step length for both posttraining volitional and FES-assisted gaits, changes were not statistically significant. Data are summarized in Table 1 for the left leg because the FES intervention was applied only to that side. The following sections compare the neurotherapeutic and neuroprosthetic results for each of the outcome measures individually.

\section{Walking Distance and Speed}

FES-assisted gait training resulted in significant voluntary gains in walking distance and speed, and the use of FES consistently produced gains beyond maximum voluntary values, as shown in Figures $\mathbf{2}$ and 3. At baseline, volitional 6-minute walk distance was $28.0 \pm 8.7 \mathrm{~m}$, which significantly increased to $80.1 \pm 2.3 \mathrm{~m}$ after gait training $(p<0.001)$, indicating a strong neurotherapeutic effect (Figure 2). Use of FES as a neuroprosthesis resulted in a significant further increase in the 6-minute walk distance to $96.5 \pm 2.9 \mathrm{~m}(p<0.001)$. Voluntary maximum walk distance increased from $33.90 \pm 16.57 \mathrm{~m}$ to $80.08 \pm 2.54 \mathrm{~m}$ at the end of the treatment phase, which trended toward statistical significance ( $p=0.008$ ) (Figure 2). However, maximum walk distance with FES greatly exceeded posttreatment values and increased significantly to $248.18 \pm 43.72 \mathrm{~m}(p<0.001)$, representing typical limited community ambulation distances and indicating a strong neuroprosthetic effect.
Speed during the volitional 6-minute walk significantly increased from a baseline value of $0.17 \pm 0.02 \mathrm{~m} / \mathrm{s}$ to $0.22 \pm$ $0.00 \mathrm{~m} / \mathrm{s}$ after gait training $(p<0.001)$ (Figure 3 ). An additional gain in speed during the FES-assisted 6-minute walk to $0.27 \pm 0.01 \mathrm{~m} / \mathrm{s}(p<0.001)$ indicated a neuroprosthetic effect of almost 20 percent. Similarly, speed during the volitional maximum walk increased significantly from a baseline value of $0.07 \pm 0.02 \mathrm{~m} / \mathrm{s}$ to $0.22 \pm 0.01 \mathrm{~m} / \mathrm{s}$ after training ( $p<0.001$ ) (Figure 3). A further gain in speed during the maximum walk to $0.26 \pm 0.01 \mathrm{~m} / \mathrm{s}$ occurred when FES was used, which was also statistically significant $(p<$ 0.001 ). Thus, gait training produced significant therapeutic effects in terms of 6-minute and maximum walk distances and speeds, which were consistently exceeded by the neuroprosthetic application of FES.

\section{Quantitative Gait Analysis}

Significant improvements in spatial-temporal gait parameters resulted from both gait training with FES and application of FES as a neuroprosthesis. Double support time during the baseline volitional data collection was $3.24 \pm 0.45 \mathrm{~s}$, which significantly decreased to $2.34 \pm 0.24 \mathrm{~s}$ after training ( $p<0.001)$ (Figure 4). Double support time further decreased to $1.81 \pm 0.09 \mathrm{~s}(p<0.001)$ during FESassisted gait, indicating a more dynamic gait.

No therapeutic or prosthetic effect was clearly demonstrated for step length. Step length did not increase significantly from baseline, although the volitional posttraining value of $0.52 \pm 0.10 \mathrm{~m}$ trended toward statistical significance relative to the baseline value of $0.36 \pm 0.06 \mathrm{~m}(p=$ 0.012). During FES-assisted gait, the step length marginally increased to $0.55 \pm 0.04 \mathrm{~m}$, which was comparable to the volitional posttraining value ( $p=0.641)$.

Table 1.

Summary of key outcome measures on left side for baseline volitional, posttraining volitional, and FES-assisted conditions (mean \pm SD).

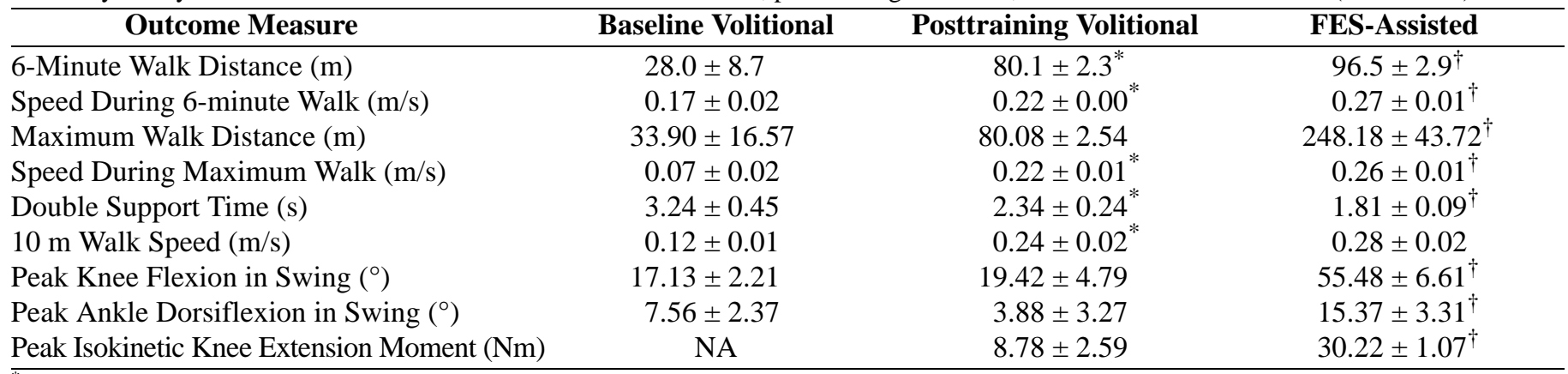

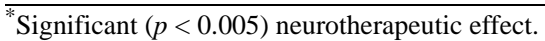

${ }^{\dagger}$ Significant $(p<0.005)$ neuroprosthetic effect.

FES = functional electrical stimulation, NA = not applicable, SD = standard deviation. 


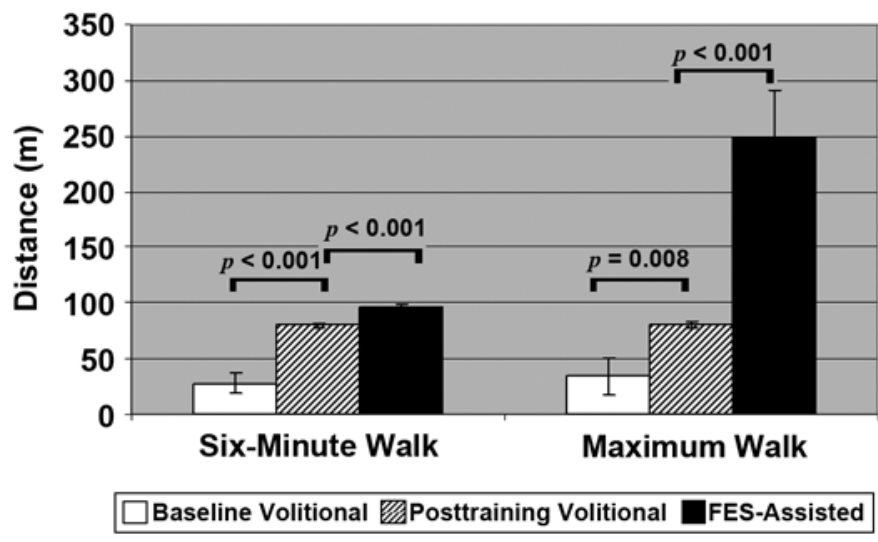

Figure 2.

Volitional 6-minute walk and maximum walk distances improved posttraining compared with baseline; distance additionally improved with functional electrical stimulation (FES).

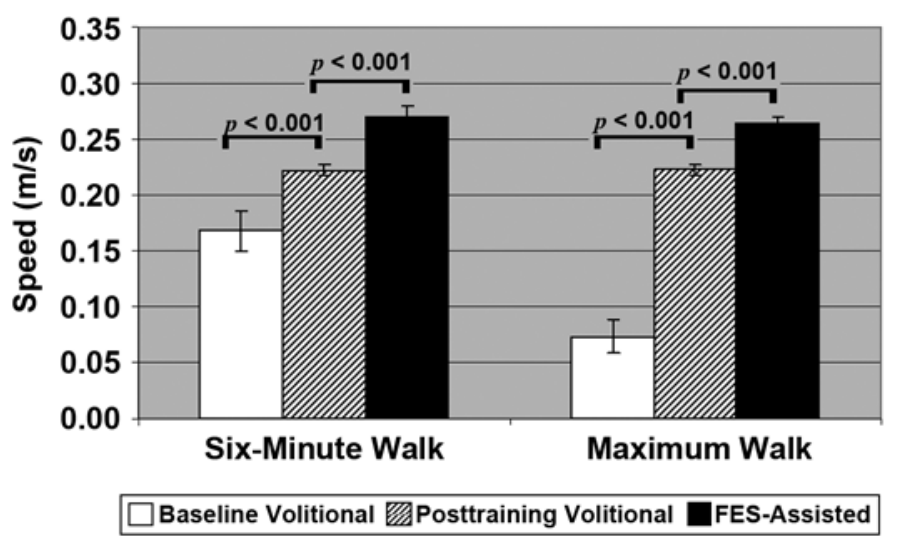

Figure 3.

Six-minute walk and maximum walk speeds improved posttraining compared with baseline volitional; speed additionally improved with functional electrical stimulation (FES).

The decrease in double support time and the trend toward increased step length led to a significant $(p<$ 0.001 ) increase in volitional $10 \mathrm{~m}$ walking speed from an initial value of $0.12 \pm 0.01 \mathrm{~m} / \mathrm{s}$ to $0.24 \pm 0.02 \mathrm{~m} / \mathrm{s}$ after training (Figure 4). This walking speed slightly increased during FES-assisted gait with a $10 \mathrm{~m}$ walking speed of $0.28 \pm 0.02 \mathrm{~m} / \mathrm{s}$, although this was not statistically different from the posttraining volitional value ( $p=0.055)$.

The kinematic parameters of volitional peak knee flexion in swing and peak ankle dorsiflexion in swing did not show statistically significant changes following rehabilitation (Figure 5). Baseline volitional peak knee flexion in
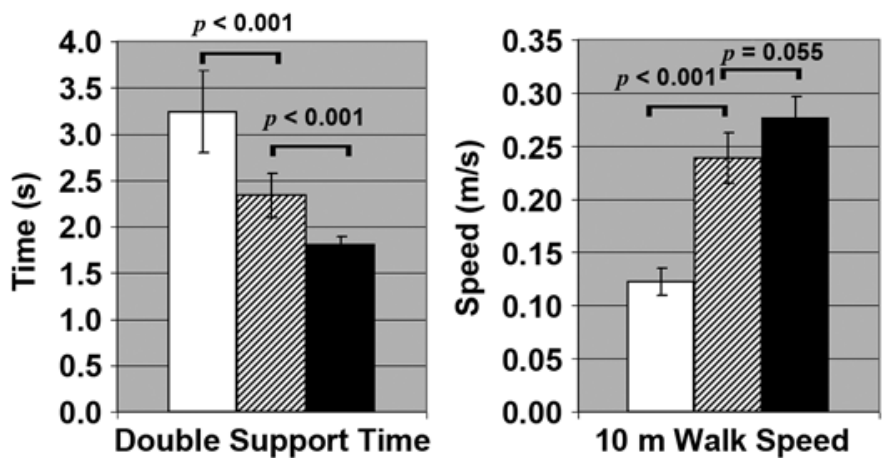

Baseline Volitional $\mathbb{Z}$ Posttraining Volitional

FES-Assisted

Figure 4.

From baseline to posttraining, volitional double support time decreased, with further reduction with functional electrical stimulation (FES); and volitional $10 \mathrm{~m}$ walking speed increased, with further gains with FES.

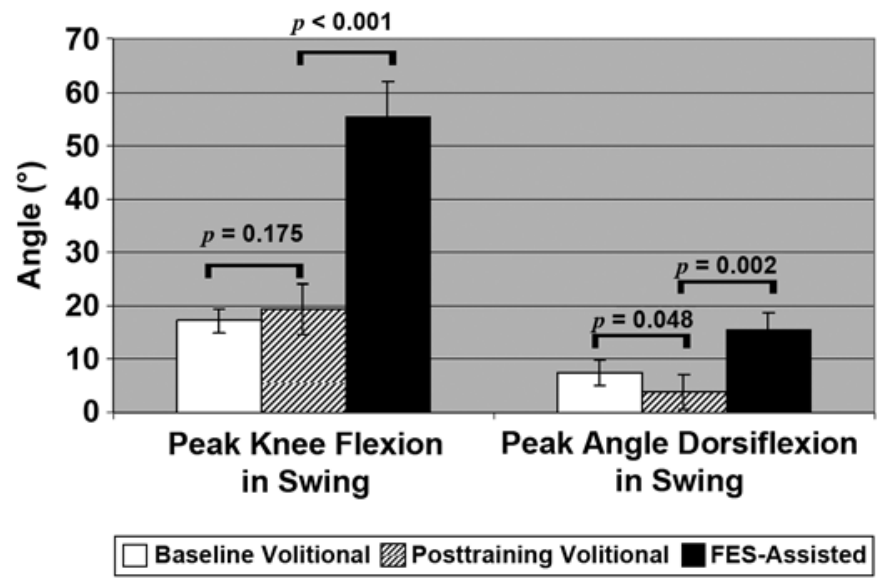

Figure 5.

Baseline and posttraining volitional peak knee flexion in swing comparable, but increase seen with functional electrical stimulation (FES). Peak ankle dorsiflexion in swing showed minor decrease from baseline to posttraining volitional but increased with FES.

swing was $17.13^{\circ} \pm 2.21^{\circ}$, which increased marginally to $19.42^{\circ} \pm 4.79^{\circ}$ after gait training ( $p=0.175$ ), while volitional peak ankle dorsiflexion in swing decrease slightly from $7.56^{\circ} \pm 2.37^{\circ}$ at baseline to $3.88^{\circ} \pm 3.27^{\circ}$ posttraining $(p=0.048)$. However, FES-assisted peak knee flexion in swing increased significantly to $55.48^{\circ} \pm 6.61^{\circ}$ ( $p<$ 0.001 ), more than doubling the posttraining volitional value. Similarly, FES-assisted peak ankle dorsiflexion in swing increased to $15.37^{\circ} \pm 3.31^{\circ}(p=0.002)$. 
The subject could not perform consistent, isolated knee extension while sitting before receiving the implanted FES system. Therefore, baseline peak isokinetic knee extension moment was not measurable. After using the FES system for exercise and ambulation, the subject could generate $8.78 \pm 2.59 \mathrm{Nm}$ of knee extension moment volitionally on the implanted side (Figure 6). When using FES to augment voluntary knee extension, the subject's peak isokinetic knee extension moment significantly increased to $30.22 \pm 1.07 \mathrm{Nm}(p<0.001)$.

Volitional ASIA motor scores derived from the manual muscle test values showed a minor increase from 5 at baseline to 6 posttraining, out of a maximum score of 25 (Table 2). FES provided a means to generate contractions that resulted in a motor score of 15 , attributable to increased hip flexion, knee extension, and ankle dorsiflexion output with stimulation. No stimulation was provided to the long

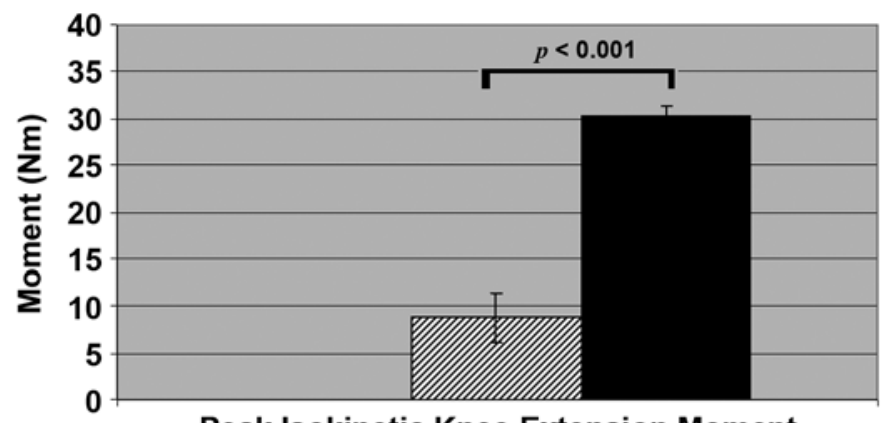

\section{Peak Isokinetic Knee Extension Moment}

Baseline Volitional $\mathbb{Z}$ Posttraining Volitional DFE-Assisted

Figure 6.

Peak isokinetic knee extension moment could not be measured at baseline, but with functional electrical stimulation (FES) was more than three times greater than at posttraining.

Table 2.

Manual muscle test scores on left side muscle groups for baseline volitional, posttraining volitional, and FES-assisted conditions. ${ }^{*}$

\begin{tabular}{lccc}
\hline \multicolumn{1}{c}{ Muscle Group } & $\begin{array}{c}\text { Baseline } \\
\text { Volitional }\end{array}$ & $\begin{array}{c}\text { Posttraining } \\
\text { Volitional }\end{array}$ & $\begin{array}{c}\text { FES- } \\
\text { Assisted }\end{array}$ \\
\hline Hip Flexors & 0 & 1 & 3 \\
Knee Extensors & 2 & 2 & 4 \\
Ankle Dorsiflexors & 0 & 0 & 5 \\
Long Toe Extensors & 0 & 0 & $0^{*}$ \\
Ankle Plantar Flexors & 3 & 3 & $3^{*}$ \\
Total Score & $\mathbf{5}$ & $\mathbf{6}$ & $\mathbf{1 5}$ \\
${ }^{*}$ No stimulation provided to long toe extensors or to ankle plantar flexors. \\
FES = functional electrical stimulation. \\
\hline \hline
\end{tabular}

toe extensors or ankle plantar flexors, which were also included in the manual muscle test score.

\section{DISCUSSION}

When comparing volitional effort pretraining with volitional effort posttraining, we saw significant $(p<0.005)$ improvements in 6-minute walk distance and speed, speed during maximum walk, double support time, and $10 \mathrm{~m}$ walking speed, demonstrating the neurotherapeutic effects of gait training with the implanted FES system. Although the step length change on the stimulated leg was meaningful, it was not statistically significant because of the conservative Bonferroni correction. The improvements in double support time and step length were consistent with the changes that would be expected in normal walking with increased speed [18].

While gait training with FES clearly resulted in volitional gains, separating the individual effects of gait training itself from the incremental effects of incorporating FES into the rehabilitation program is impossible. While stimulation possibly could have accelerated the pace or amplified the extent of volitional gains that could have been made by conventional gait training alone, the subject could also possibly have achieved the same therapeutic effects from conventional gait training without FES. The differential effects of gait training with FES versus conventional therapy methods on voluntary function remains to be determined in future studies. However, the results of this investigation strongly suggest that the combination of FES with gait training may be an effective neurotherapeutic tool for improving volitional function in ambulatory individuals with chronic incomplete SCI.

The increased knee extension moment and small improvement in manual muscle test scores demonstrated that an increase in strength of the stimulated muscles occurred in addition to improved performance during walking. Although the volitional manual muscle test score showed little improvement, the manual muscle test score with FES improved markedly because stimulation provided a means to augment voluntary muscle force and improve the output of hip flexion, knee extension, and ankle dorsiflexion torques.

Posttraining, FES-assisted gait showed significant $(p<$ 0.005 ) improvements over volitional effort. Significant neuroprosthetic effects above maximum volitional effort were observed for 6-minute walk distance and speed, maximum 
walk distance and speed, double support time, peak knee flexion in swing, peak ankle dorsiflexion in swing, and knee extension moment. These gains demonstrated how an implanted FES system can improve ambulation for a person with incomplete SCI beyond the neurotherapeutic effects of gait training. Use of the implanted system generally resulted in improvements approximately 20 percent over maximum voluntary function in the selected metrics of gait performance.

An increase in peak knee flexion during swing occurred posttraining when the subject walked using FES even though no stimulation was given to the hamstrings for knee flexion. Most likely the increased knee flexion during swing was a result of stimulation to the hip flexors and ankle dorsiflexors, causing a flexor-synergy or flexionwithdraw pattern of the entire limb. Another possibility is that the knee flexors were activated by an overflow of stimulation from another electrode.
The magnitude and value of the neuroprosthetic effect of the implanted FES system is most apparent by comparing the 6-minute and maximum walk distances. After training, the volitional 6-minute and maximum walk distances were both approximately $80 \mathrm{~m}$, indicating that maximal voluntary function was roughly equivalent to that which could be covered in 6 minutes. FES-assisted 6-minute walk distance was significantly greater than maximum voluntary effort but still modest at $96 \mathrm{~m}$. However, the FES-assisted maximum walk distance was $248 \mathrm{~m}$. This threefold improvement was possible only through the neuroprosthetic use of FES and demonstrates the advantage of walking with the implanted system over volitional function alone. With the capability to walk such distances, a person with incomplete SCI could possibly perform limited community ambulation activities with FES-assistance (Figure 7).

Generalizing the results of this single-subject study to the larger and extremely heterogeneous incomplete SCI population may be difficult. Both the neurotherapeutic and

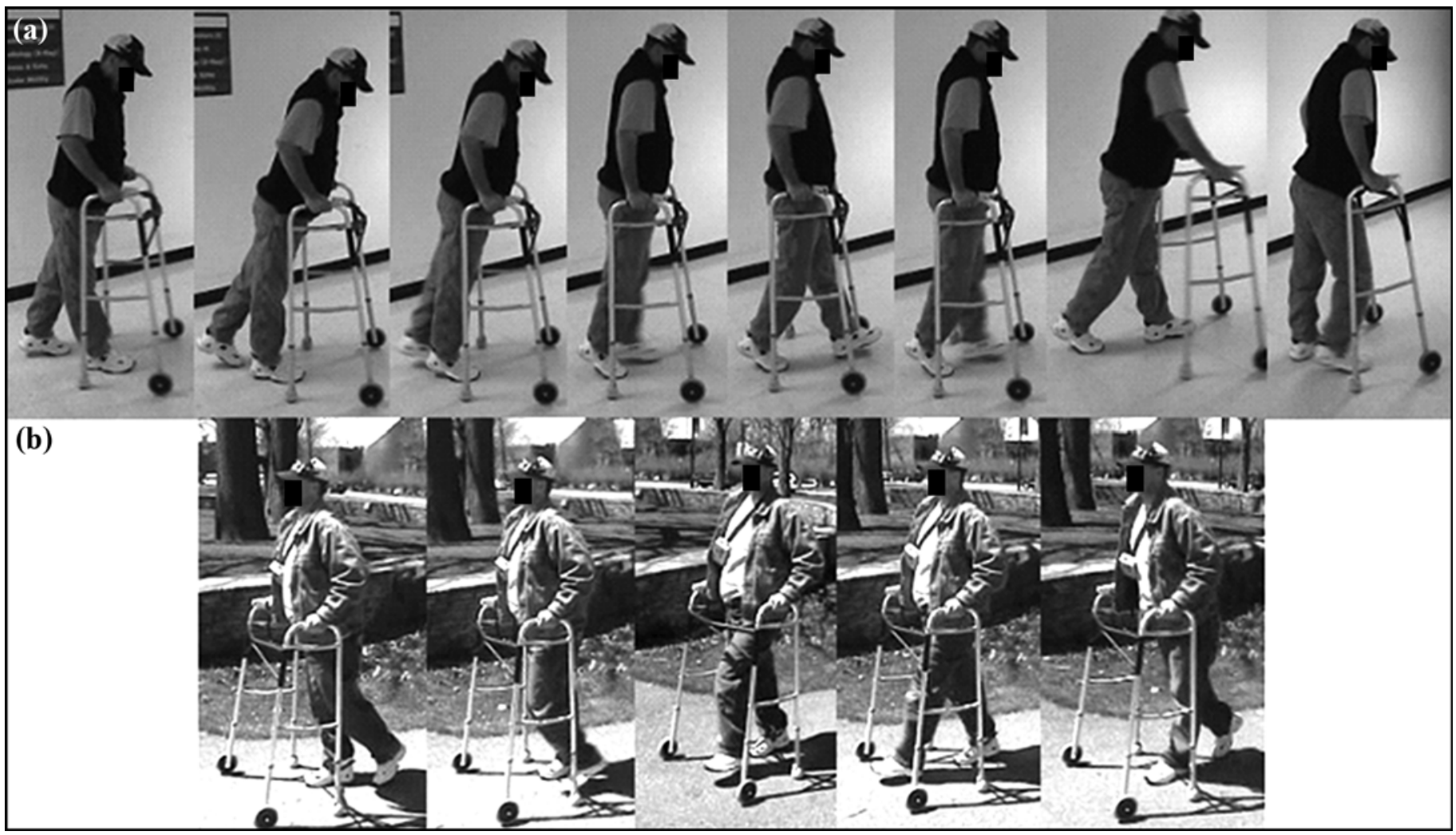

Figure 7.

Voluntary and functional electrical stimulation (FES)-assisted ambulatory performance: (a) Baseline volitional walking limited primarily by upper-limb fatigue due to compensatory strategy requiring subject to elevate body to clear weaker leg during swing phase. (b) FES assistance addressed major swing phase deficits and enabled subject to perform limited community ambulation activities. Click Here to Play Videos 
neuroprosthetic effects of FES can be expected to vary greatly, depending on the severity of motor and sensory loss and the level of injury. The neurological, physical, and psychological characteristics of individuals with incomplete SCI who may benefit most from FES-assisted ambulation should be a focus of future studies. While gains in both volitional and FES-assisted walking function were significant, they were still modest compared with nondisabled norms. Optimizing the therapeutic and prosthetic effects of FES to restore ambulatory ability as close as possible to normal values is also an area for further research.

When walking with FES, the subject did not need to wear his left AFO, which increased convenience and simplified walking readiness. However, he still needed a wheeled walker to compensate for the lack of trunk and hip stability. At the end of the study, poor lower-limb timing and coordination on the non-FES side, including lack of mediolateral hip stability, became the primary factors that limited walking ability. These issues became apparent as walking speed increased during training.

Subjective ratings of user effort and satisfaction with voluntary and FES-assisted walking were collected regularly during the study, although not frequently enough for rigorous statistical analysis. The Usability Rating Scale (URS), an established method for discovering subjective impressions of the relative ease or difficulty in performing life tasks after assistive technology intervention [19-20], was applied after several walking sessions with and without FES throughout baseline testing, gait training, and posttraining data collection sessions. The URS is a 7-point ordinal scale in which a user can rate an activity from very difficult $(-3)$ to very easy $(+3)$. At the start of training with the implanted FES system, the participant rated walking with FES as barely difficult $(-1)$ and still held a preference for volitional walking (moderately easy, +2). After completing training with the implanted FES system, the participant rated walking with and without FES at equivalent levels of difficulty (both moderately easy, +2). Walking with FES was difficult for the participant initially, because he had to learn to coordinate his non-FES limb with the stimulated limb for walking. As training progressed, this process was improved and then made walking with FES similar to walking volitionally.

In this study, patterns of stimulation for exercise and ambulation were selected from a menu of options via a button on the external control unit or with a finger switch. In the future, triggering FES patterns could be accomplished via more natural commands, such as volitional electromyo- graphy from recording electrodes inserted into muscles under voluntary control that are associated with ambulation. Using a natural command source to control the neuroprosthesis would be particularly useful for persons with cervical-level incomplete injuries, the largest subgroup of the SCI population, because they might exhibit upper-limb impairment that would preclude the use of manual switches or buttons to control stimulation timing.

\section{CONCLUSIONS}

This single-subject study quantified both the therapeutic effects of incorporating an implanted FES system into a gait rehabilitation program for an ambulatory individual with chronic incomplete SCI, as well as the prosthetic effects of using FES during walking. Gait training with the implanted FES system resulted in improvements in volitional function over baseline values, demonstrating a significant $(p<0.005)$ neurotherapeutic effect on (1) 6-minute walk distance, (2) speed during 6-minute walk, (3) speed during maximum walk, (4) double support time, and (5) $10 \mathrm{~m}$ walking speed. These changes can be attributed to enhanced voluntary strength and coordination derived from 36 sessions of FESassisted gait training.

Walking performance was almost uniformly improved beyond maximal voluntary function by the application of FES during walking. When compared with maximal voluntary performance, FES-assisted gait produced additional and significant $(p<0.005)$ neuroprosthetic improvements in (1) 6-minute walk distance, (2) speed during 6-minute walk, (3) maximum walk distance, (4) speed during maximum walk, (5) peak knee flexion in swing, (6) peak ankle dorsiflexion in swing, and (7) peak isokinetic knee extension moment. The increased distance, speed, joint angles, and strength attributable to the use of the implanted FES system may enable individuals with incomplete SCI to become limited community ambulators. The neurotherapeutic benefits of gait training with FES and the neuroprosthetic effects of FES-assisted gait to an ambulatory individual with long-standing chronic incomplete SCI have been shown to significantly improve gait performance in this single-subject case study.

\section{ACKNOWLEDGMENTS}

Author Contributions:

Study concept and design: R. J. Triolo. 
Acquisition of data: L. M. Boggs, S. N. Bailey.

Analysis and interpretation of data: R. J. Triolo, E. C. Hardin,

S. N. Bailey.

Drafting of manuscript: S. N. Bailey.

Critical revision of manuscript for important intellectual content:

R. J. Triolo, R. Kobetic, E. C. Hardin, L. M. Boggs.

Statistical analysis: S. N. Bailey, E. C. Hardin.

Obtained funding: R. J. Triolo, G. Pinault.

Technical, clinical, or material support: L. M. Boggs, R. Kobetic, G. Pinault.

Study supervision: R. J. Triolo.

Financial Disclosures: The authors declared that no competing interests exist.

Funding/Support: This material is based on work supported by the VA Rehabilitation Research and Development Service, merit review B2933R (Triolo).

Additional Contributions: We acknowledge the support of merit review grant B2933R from the VA Rehabilitation Research and Development Service and the many contributions of the research volunteer in this single-subject case study. Portions of this material were presented at the 2007 Annual Meeting of the International Function Electrical Stimulation Society, held November 10-14, 2007, in Philadelphia, Pennsylvania.

Institutional Review: This study was approved by the Louis Stokes VA Medical Center Institutional Review Board.

Participant Follow-Up: The authors plan to inform the participant of the publication of this study.

\section{REFERENCES}

1. Sekhon LH, Fehlings MG. Epidemiology, demographics, and pathophysiology of acute spinal cord injury. Spine. 2001;26(24 Suppl):S2-12. [PMID: 11805601]

DOI:10.1097/00007632-200112151-00002

2. Berkowitz M. The economic consequences of traumatic spinal cord injury. New York (NY): Demos; 1992.

3. Dobkin B, Barbeau H, Deforge D, Ditunno J, Elashoff R, Apple D, Basso M, Behrman A, Harkema S, Saulino M, Scott M; Spinal Cord Injury Locomotor Trial Group. The evolution of walking-related outcomes over the first 12 weeks of rehabilitation for incomplete traumatic spinal cord injury: The multicenter randomized Spinal Cord Injury Locomotor Trial. Neurorehabil Neural Repair. 2007; 21(1):25-35. [PMID: 17172551]

DOI:10.1177/1545968306295556

4. Postans NJ, Hasler JP, Granat MH, Maxwell DJ. Functional electric stimulation to augment partial weight-bearing supported treadmill training for patients with acute incomplete spinal cord injury: A pilot study. Arch Phys Med Rehabil. 2004;85(4):604-10. [PMID: 15083437]

DOI:10.1016/j.apmr.2003.08.083

5. Granat MH, Ferguson AC, Andrews BJ, Delargy M. The role of functional electrical stimulation in the rehabilitation of patients with incomplete spinal cord injury-Observed benefits during gait studies. Paraplegia. 1993;31(4):207-15. [PMID: 8493035]

6. Field-Fote EC. Combined use of body weight support, functional electrical stimulation, and treadmill training to improve walking ability in individuals with chronic incomplete spinal cord injury. Arch Phys Med Rehabil. 2001; 82(6):818-24. [PMID: 11387589]

DOI:10.1053/apmr.2001.23752

7. Thrasher TA, Flett HM, Popovic MR. Gait training regimen for incomplete spinal cord injury using functional electrical stimulation. Spinal Cord. 2006;44(6):357-61. [PMID: 16249784] DOI:10.1038/sj.sc.3101864

8. Field-Fote EC, Lindley SD, Sherman AL. Locomotor training approaches for individuals with spinal cord injury: A preliminary report of walking-related outcomes. J Neurol Phys Ther. 2005;29(3):127-37. [PMID: 16398945]

9. Wieler M, Stein RB, Ladouceur M, Whittaker M, Smith AW, Naaman S, Barbeau H, Bugaresti J, Aimone E. Multicenter evaluation of electrical stimulation systems for walking. Arch Phys Med Rehabil. 1999:80(5):495-500. [PMID: 10326910] DOI:10.1016/S0003-9993(99)90188-0

10. Bonaroti D, Akers JM, Smith BT, Mulcahey MJ, Betz RR. Comparison of functional electrical stimulation to long leg braces for upright mobility for children with complete thoracic level spinal injuries. Arch Phys Med Rehabil. 1999; 80(9):1047-53. [PMID: 10489007]

DOI:10.1016/S0003-9993(99)90059-X

11. Johnston TE, Betz RR, Smith BT, Mulcahey MJ. Implanted functional electrical stimulation: An alternative for standing and walking in pediatric spinal cord injury. Spinal Cord. 2003;41(3):144-52. [PMID: 12612616] DOI:10.1038/sj.sc.3101392

12. Kobetic R, Triolo RJ, Uhlir JP, Bieri C, Wibowo M, Polando G, Marsolais EB, Davis JA Jr, Ferguson KA. Implanted functional electrical stimulation system for mobility in paraplegia: A follow-up case report. IEEE Trans Rehabil Eng. 1999;7(4):390-98. [PMID: 10609626$]$ DOI:10.1109/86.808942

13. Hardin E, Kobetic R, Murray L, Corado-Ahmed M, Pinault G, Sakai J, Bailey SN, Ho C, Triolo RJ. Walking after incomplete spinal cord injury using an implanted FES system: A case report. J Rehabil Res Dev. 2007;44(3):333-46. [PMID: 18247230]

DOI:10.1682/JRRD.2007.03.0333

14. Davis JA Jr, Triolo RJ, Uhlir JP, Bhadra N, Lissy DA, Nandurkar S, Marsolais EB. Surgical technique for installing an eight-channel neuroprosthesis for standing. Clin Orthop Relat Res. 2001;(385):237-52. [PMID: 11302320] DOI:10.1097/00003086-200104000-00035 
JRRD, Volume 47, Number 1, 2010

15. Davis JA Jr, Triolo RJ, Uhlir J, Bieri C, Rohde L, Lissy D, Kukke S. Preliminary performance of a surgically implanted neuroprosthesis for standing and transfers-Where do we stand? J Rehabil Res Dev. 2001;38(6):609-17. [PMID: 11767968]

16. Buckett J, Triolo R, Ferencz D, Katorgi M, Bieri C. A wearable controller for clinical studies involving multiimplant FNS systems. J Spinal Cord Med. 1998;21(2):179.

17. Vicon plug-in gait product guide-Foundation notes. Revision 1.0. Los Angeles (CA): Vicon; 2008. p. 11-12.

18. Perry J. Gait analysis: Normal and pathological function. Thorofare (NJ): Slack; 1992.
19. Steinfeld E, Danford GS. Enabling environments: Measuring the impact of environment on disability and rehabilitation. New York (NY): Kluwer Academic/Plenum Publishers; 1999.

20. Dittmar SS, Gresham GE. Functional assessment and outcome measures for the rehabilitation health professional. Gaithersburg (MD): Aspen Publishers; 1997.

Submitted for publication March 27, 2009. Accepted in revised form October 5, 2009. 